CRYSTALLOGRAPHIC COMMUNICATIONS

ISSN 2056-9890

Received 20 September 2015

Accepted 23 September 2015

Edited by M. Zeller, Youngstown State University, USA

Keywords: crystal structure; hydrogen-bond framework; polymorph

Supporting information: this article has supporting information at journals.iucr.org/e
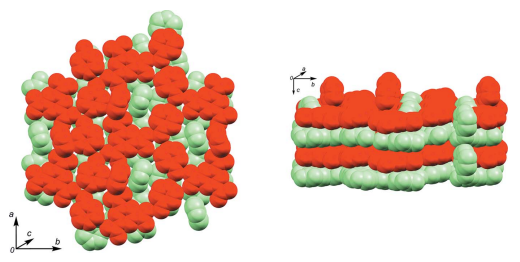

OPEN $\odot$ ACCESS

\section{Crystal structure of benzene-1,3,5-tricarboxylic acid-4-pyridone (1/3)}

\author{
Selena L. Staun and Allen G. Oliver*
}

Department of Chemistry and Biochemistry, University of Notre Dame, Notre Dame, IN 46556-5670, USA. *Correspondence e-mail: aoliver2@nd.edu

Slow co-crystallization of a solution of benzene-1,3,5-tricarboxylic acid with a large excess of 4-hydroxypyridine produces an interpenetrating, three-dimensional, hydrogen-bonded framework consisting of three 4-pyridone and one benzene-1,3,5-tricarboxylic acid molecules, $\mathrm{C}_{9} \mathrm{H}_{6} \mathrm{O}_{6} \cdot 3 \mathrm{C}_{5} \mathrm{H}_{5} \mathrm{NO}$. This structure represents an orthorhombic polymorph of the previously reported $C$-centered, monoclinic structure [Campos-Gaxiola et al. (2014). Acta Cryst. E70, o453o454].

\section{Chemical context}

We have been interested in the co-crystallization properties of benzene carboxylic acid derivatives (namely: benzene-1,4-dicarboxylic acid and benzene-1,3,5-tricarboxylic acid) with 3and 4-hydroxypyridines (Staun \& Oliver, 2012, 2015; Bhogala et al., 2005). A variety of 3-hydroxypyridine co-crystallants with benzene carboxylic acids have already been reported and we discontinued pursuit of those materials (Shattock et al., 2008). Both 4-hydroxypyridine and benzene-1,3,5-tricarboxylic acid have been used extensively in both metal-organic frameworks as well as suitable donor/acceptor species in crystal engineering (see for example: Castillo et al., 2001; Qian et al., 2014). Recently we reported the characterization of the 1:1 co-crystallant 4-hydroxypyridinium 3,5-dicarboxybenzoate (Staun \& Oliver, 2015). We also discovered that from similar preparative conditions (slow evaporation from methanol) with a larger molar ratio of 4-hydroxypyridine to benzene-1,3,5tricarboxylic acid (BTC) a new species could be obtained; reported herein. A comparison of the structure with the Cambridge Structure Database revealed an identical structural motif, albeit in a different crystal system (CamposGaxiola et al., 2014). Thus, we report the orthorhombic polymorph of benzene-1,3,5-tricarboxylic acid-4-pyridone (1/3).

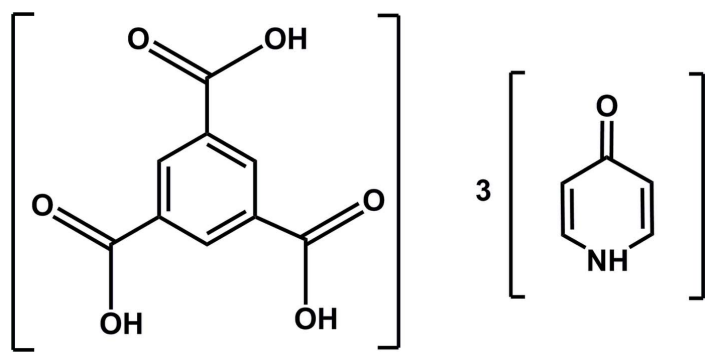

\section{Structural commentary}

The dihedral angles formed by the carboxylic acid moieties with respect to the benzene ring are 2.95 (16), 6.23 (10) and 
Figure 1

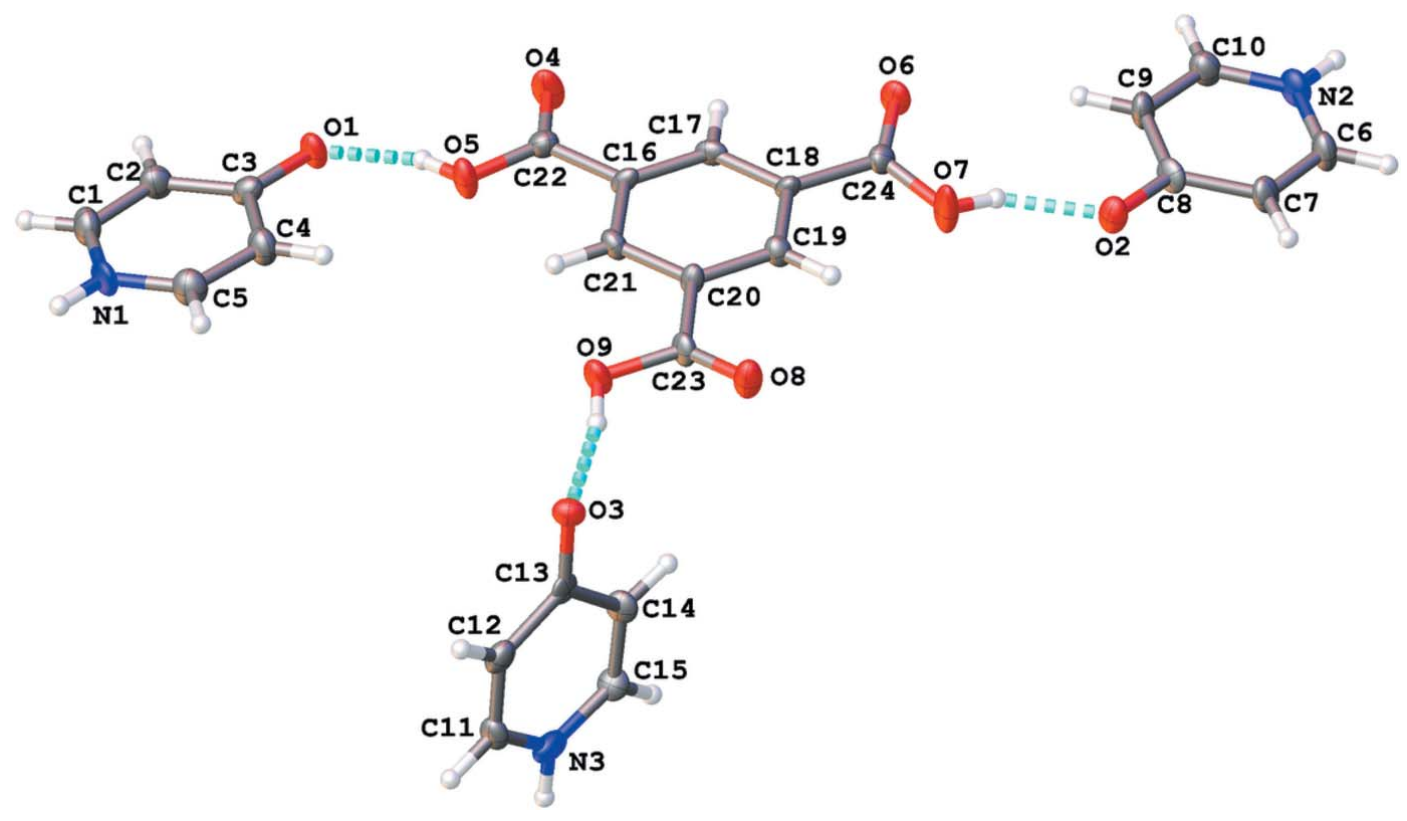

Labeling scheme for title compound. Atomic displacement ellipsoids are depicted at the $50 \%$ probability level. Dashed lines represent hydrogen bonds within the asymmetric unit.

$10.28(18)^{\circ}$. These are comparable with those for the previously reported polymorph of this compound [3.9 (2), 9.3 (2), and 13.3 (2) ${ }^{\circ}$; Campos-Gaxiola et al., 2014]. It should be noted that the 4-hydroxypyridine has undergone rearrangement from a hydroxypyridine to the pyridone form of the

Table 1

Pyridone / BTC interplanar angles $\left(^{\circ}\right)$.

\begin{tabular}{lll}
\hline Pyridone ring & This work & Campos-Gaxiola \\
\hline N1 & $7.3(2)$ & 12.9 \\
N2 & $8.5(2)$ & 13.2 \\
N3 & $87.5(3)$ & 87.1 \\
\hline
\end{tabular}

Table 2

Hydrogen-bond geometry $\left(\AA,^{\circ}\right)$.

\begin{tabular}{|c|c|c|c|c|}
\hline$D-\mathrm{H} \cdots A$ & $D-\mathrm{H}$ & $\mathrm{H} \cdots A$ & $D \cdots A$ & $D-\mathrm{H} \cdots A$ \\
\hline $\mathrm{N} 1-\mathrm{H} 1 N \cdots \mathrm{O} 1^{\mathrm{i}}$ & 0.88 & 1.89 & $2.762(8)$ & 169 \\
\hline $\mathrm{N} 2-\mathrm{H} 2 N \cdots \mathrm{O} 2^{\mathrm{ii}}$ & 0.88 & 1.90 & $2.711(8)$ & 152 \\
\hline $\mathrm{N} 3-\mathrm{H} 3 N \cdots \mathrm{O} 3^{\mathrm{iii}}$ & 0.88 & 2.01 & $2.773(10)$ & 144 \\
\hline $\mathrm{N} 3-\mathrm{H} 3 N \cdots \mathrm{O}^{\mathrm{iv}}$ & 0.88 & 2.59 & $3.124(9)$ & 120 \\
\hline $\mathrm{O} 5-\mathrm{H} 5 O \cdots \mathrm{O} 1$ & 0.84 & 1.75 & $2.555(7)$ & 161 \\
\hline $\mathrm{O} 7-\mathrm{H} 70 \cdots \mathrm{O} 2$ & 0.84 & 1.73 & $2.463(7)$ & 145 \\
\hline $\mathrm{O} 9-\mathrm{H} 9 O \cdots \mathrm{O} 3$ & 0.84 & 1.70 & $2.526(7)$ & 167 \\
\hline $\mathrm{C} 1-\mathrm{H} 1 \cdots \mathrm{O} 4^{\mathrm{i}}$ & 0.95 & 2.38 & $3.227(10)$ & 148 \\
\hline $\mathrm{C} 4-\mathrm{H} 4 \cdots \mathrm{O} 5$ & 0.95 & 2.53 & $3.174(9)$ & 126 \\
\hline $\mathrm{C} 6-\mathrm{H} 6 \cdots \mathrm{O} 7^{\mathrm{ii}}$ & 0.95 & 2.26 & $3.051(9)$ & 140 \\
\hline $\mathrm{C} 7-\mathrm{H} 7 \cdots \mathrm{O} 8^{\mathrm{ii}}$ & 0.95 & 2.66 & $3.530(9)$ & 153 \\
\hline $\mathrm{C} 9-\mathrm{H} 9 \cdots \mathrm{O}^{\mathrm{v}}$ & 0.95 & 2.58 & $3.227(9)$ & 126 \\
\hline $\mathrm{C} 11-\mathrm{H} 11 \cdots \mathrm{O}^{\mathrm{iv}}$ & 0.95 & 2.46 & $3.076(11)$ & 123 \\
\hline $\mathrm{C} 11-\mathrm{H} 11 \cdots \mathrm{O}^{\mathrm{vi}}$ & 0.95 & 2.55 & $3.159(9)$ & 122 \\
\hline $\mathrm{C} 12-\mathrm{H} 12 \cdots \mathrm{O}^{\mathrm{vii}}$ & 0.95 & 2.49 & $3.302(11)$ & 143 \\
\hline $\mathrm{C} 14-\mathrm{H} 13 \cdots \mathrm{O} 4^{\text {viii }}$ & 0.95 & 2.60 & $3.405(10)$ & 143 \\
\hline $\mathrm{C} 15-\mathrm{H} 15 \cdots \mathrm{O}^{\mathrm{iii}}$ & 0.95 & 2.66 & $3.608(10)$ & 178 \\
\hline
\end{tabular}

Symmetry codes: (i) $x-\frac{1}{2},-y+\frac{1}{2}, z$; (ii) $x+\frac{1}{2},-y+\frac{3}{2}, z$; (iii) $x, y, z+1$; (iv) $x-1, y, z+1 ; \quad$ (v) $\quad x+1, y, z ; \quad$ (vi) $\quad-x,-y+1, z+\frac{1}{2}$; (vii) $x-1, y, z$; (viii) $-x+1,-y+1, z+\frac{1}{2}$. molecule as previously observed (Tyl et al., 2008). The 4-pyridone $\mathrm{C}-\mathrm{O}$ bond distances range from 1.280 (8) to 1.295 (8) ^. These distances are comparable with previously reported examples of this molecule (Staun \& Oliver, 2012; Tyl et al., 2008). Inspection of the bond distances about each pyridone ring shows a slight tendency for the $\mathrm{C}-\mathrm{C}$ bonds $\alpha$ to the nitrogen [1.347 (12) to $1.371(11) \AA]$ to be shorter than those to the carbonyl carbon [1.410 (11) to 1.421 (10) A]]. This supports the proposed formal, localized double bond along the 'edges' of the pyridone ring.

Two of the three 4-pyridone rings are co-planar with the benzene tricarboxylic acid moiety, similar to that of the previously reported structure (Campos-Gaxiola et al., 2014). The remaining 4-pyridone is essentially perpendicular to this plane, also similar to the Campos-Gaxiola structure (Table 1).

\section{Supramolecular features}

Each of the pyridone molecules forms a hydrogen-bonded chain of symmetry-related molecules. N1 and $\mathrm{N} 2$ form hydrogen bonds to $\mathrm{O}_{1}{ }^{\mathrm{i}}$ and $\mathrm{O} 2^{\mathrm{ii}}$, respectively, related by the crystallographic $n$-glide [symmetry codes: (i) $x-\frac{1}{2},-y+\frac{1}{2}, z$; (ii) $\left.x+\frac{1}{2},-y+\frac{3}{2}, z\right]$. $\mathrm{N} 3$ forms hydrogen bonds to $\mathrm{O} 3{ }^{\text {iii }}$ and $\mathrm{O} 6{ }^{\text {iv }}$ related by translation along the crystallographic $c$-axis and the [101] direction, respectively [symmetry codes: (iii) $x, y, z+1$; (iv) $x-1, y, z+1)$. Thus $\mathrm{N} 3$ forms a bifurcated hydrogen bond. These chains of hydrogen-bonded pyridone molecules are bridged by the BTC molecule. Each carboxylic acid moiety on BTC donates a hydrogen bond to a nearby pyridone carbonyl oxygen (Fig.1, Table 2). These $\mathrm{O}_{\mathrm{COOH}} \cdots \mathrm{O}_{\text {py }}$ contacts are short for $\mathrm{O}-\mathrm{H} \cdots \mathrm{O}$ contacts indicating strong intermolecular hydrogen bonding. As a result of the N3 pyridone being oriented almost perpendicular to the plane of the other 


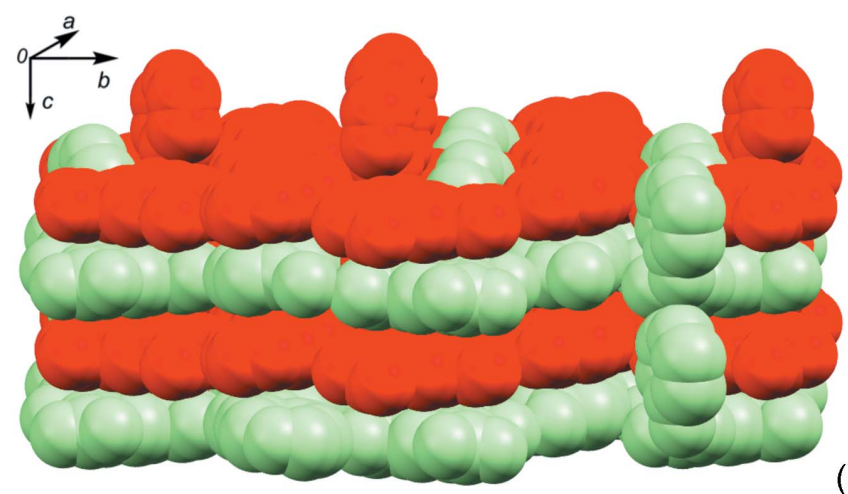

(a)

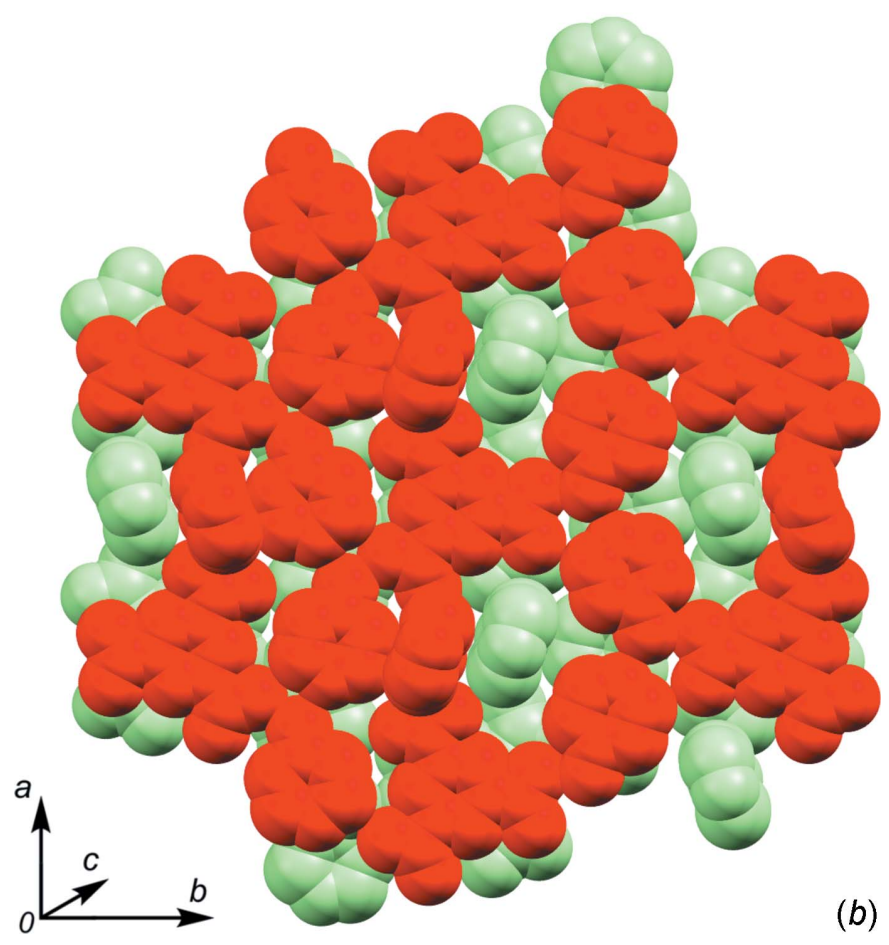

Figure 2

Space-filling views displaying the interpenetrating networks $(a)$ along the $a$ axis; $(b)$ along the $c$ axis.

three molecules, the resulting architecture is a three-dimensional hydrogen-bonded network. The BTC, N1 and N2 pyridone molecules form a graph-set $R_{8}^{6}(44)$ ring that is parallel with the $a b$ plane (Macrae et al., 2008). This corresponds with that observed by Campos-Gaxiola et al. The BTC and $\mathrm{N} 3$ pyridone form an $R_{5}^{5}(30)$ ring that is perpendicular to the previous ring. Further inspection of this network reveals that there are two independent, interpenetrating networks (Fig. 2). The BTC molecules in the two networks form typical slipped $\pi-\pi$-stacks $\left[C_{g} \cdots C_{g}=3.592(5) \AA, C_{g} \cdots\right.$ perp $=$ 3.302 (4) $\AA$; $C_{g}$ represents the center of gravity of the ring, perp is the shortest perpendicular distance; Spek, 2009]. Other potential $\pi-\pi$ contacts are beyond $4 \AA$. Due to the efficient packing of these molecules there is a significant number of close $\mathrm{C}-\mathrm{H} \cdots \mathrm{O}$ contacts, primarily between pyridone carbon atoms and carboxylic acid oxygen atoms, with one notable example being a contact from $\mathrm{C} 9$ to $\mathrm{O}^{\mathrm{v}}$ [symmetry code: (v) $x+1, y, z]$.

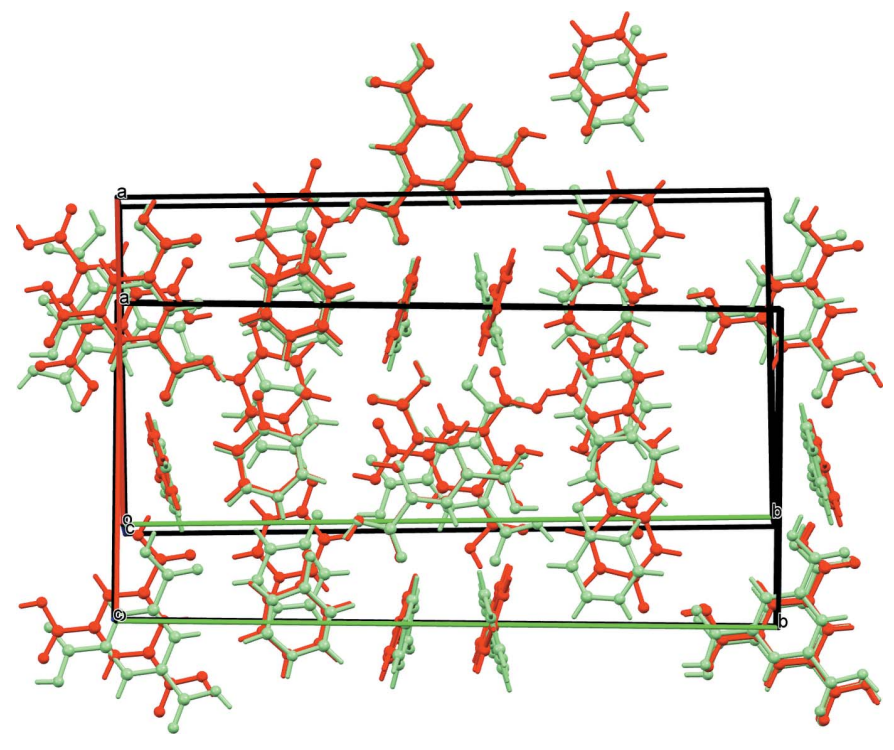

Figure 3

Overlay of the title compound (red) with the Campos-Gaxiola (light green) structure. The BTC moiety is used as the target for overlay. The view is along the $c$ axis of both structures. Non-H atoms depicted as arbitrary spheres, $\mathrm{H}$ atoms as short sticks.

\section{Database survey}

A search in the Cambridge Structural Database (CSD, Version 5.36 plus 3 updates; Groom \& Allen, 2014) for 4-hydroxypyridine with benzene-1,3,5-tricarboxylic acid produced only one hit. The compound is closely related to the title compound, namely: benzene-1,3,5-tricarboxylic acid-pyridinium-2-olate (1/3) (Campos-Gaxiola et al., 2014). However, the structure is reported to be in the monoclinic space group $C$ c.

\section{Comparison with the structure of the monoclinic polymorph}

Inspection of an overlay of the two structures reveals some differences between the two polymorphs (Fig. 3). The orientation of the carboxylic acid groups of the BTC in the title compound has one 'reversed' with respect to the others, while the Campos-Gaxiola structure has all three oriented in the same direction, forming a propeller-like motif about the BTC. This results in a change in the hydrogen-bonding motif, reversing the orientations of the pyridone moieties. Perhaps the most prominent structural change is the orientation of the pyridone perpendicular to the plane of the BTC. In the title compound the pyridone rings are oriented with planes that are parallel to each other along the channels they occupy and are related by the screw axis parallel to the $c$ axis. The perpendicular pyridone rings in the Campos-Gaxiola structure alternate their orientation along the channel, related by the $c$ glide. The change in hydrogen-bonding directionality is propagated to the orientation of the $\mathrm{N} 1$ and $\mathrm{N} 2$ pyridone chains. Examining the orientation of the carbonyl of the pyridone in these two chains reveals that the Campos-Gaxiola structure has the N1 and $\mathrm{N} 2$ chains oriented with the carbonyl 
Table 3

Experimental details.

\begin{tabular}{|c|c|}
\hline \multicolumn{2}{|l|}{ Crystal data } \\
\hline Chemical formula & $\mathrm{C}_{9} \mathrm{H}_{6} \mathrm{O}_{6} \cdot 3 \mathrm{C}_{5} \mathrm{H}_{5} \mathrm{NO}$ \\
\hline$M_{\mathrm{r}}$ & 495.44 \\
\hline Crystal system, space group & Orthorhombic, Pna $_{1}$ \\
\hline Temperature $(\mathrm{K})$ & 120 \\
\hline$a, b, c(\AA)$ & $12.699(3), 26.498(6), 6.6591(14)$ \\
\hline$V\left(\AA^{3}\right)$ & $2240.9(8)$ \\
\hline$Z$ & 4 \\
\hline Radiation type & Мо $K \alpha$ \\
\hline$\mu\left(\mathrm{mm}^{-1}\right)$ & 0.11 \\
\hline Crystal size (mm) & $0.11 \times 0.07 \times 0.05$ \\
\hline \multicolumn{2}{|l|}{ Data collection } \\
\hline Diffractometer & Bruker APEXII \\
\hline Absorption correction & $\begin{array}{l}\text { Multi-scan (SADABS; Krause et } \\
\quad \text { al., 2015) }\end{array}$ \\
\hline$T_{\min }, T_{\max }$ & $0.647,0.745$ \\
\hline $\begin{array}{l}\text { No. of measured, independent and } \\
\text { observed }[I>2 \sigma(I)] \text { reflections }\end{array}$ & $19034,3257,2418$ \\
\hline$R_{\text {int }}$ & 0.109 \\
\hline$\theta_{\max }\left({ }^{\circ}\right)$ & 23.4 \\
\hline$(\sin \theta / \lambda)_{\max }\left(\AA^{-1}\right)$ & 0.558 \\
\hline \multicolumn{2}{|l|}{ Refinement } \\
\hline$R\left[F^{2}>2 \sigma\left(F^{2}\right)\right], w R\left(F^{2}\right), S$ & $0.069,0.171,1.04$ \\
\hline No. of reflections & 3257 \\
\hline No. of parameters & 328 \\
\hline No. of restraints & 1 \\
\hline $\mathrm{H}$-atom treatment & $\mathrm{H}$-atom parameters constrained \\
\hline$\Delta \rho_{\max }, \Delta \rho_{\min }\left(\mathrm{e} \AA^{-3}\right)$ & $0.43,-0.43$ \\
\hline
\end{tabular}

Computer programs: APEX2 and SAINT (Bruker 2012), SHELXS97 (Sheldrick, 2008), SHELXL2014/7 (Sheldrick, 2015), OLEX2 (Dolomanov et al., 2009), Mercury (Macrae et al., 2008) and publCIF (Westrip, 2010).

along the $a$-axis forming a 'parallel' alignment of the adjacent pyridone chains; again the $c$-glide is the cause for this arrangement. The $\mathrm{N} 1$ and $\mathrm{N} 2$ chains in the title compound adopt an 'anti-parallel' orientation with carbonyls in one chain being oriented in the opposite direction to the next chain, again a function of the screw axis. This is highlighted in Fig. 3 with the pyridone chain on the left of the figure showing an overlap of the pyridone rings between the two structures and the chain on the right of the figure showing the opposite orientation of the pyridone rings.

\section{Synthesis and crystallization}

The compound was formed by dissolving 4-hydroxypyridine $(0.112 \mathrm{~g}, 1.18 \mathrm{mmol})$ in methanol $(3 \mathrm{~mL})$ and benzene $1,3,5-$ tricarboxylic acid $(0.052 \mathrm{~g}, 0.24 \mathrm{mmol})$ in methanol $(3 \mathrm{~mL})$. The two solutions were combined and allowed to evaporate over $5 \mathrm{~d}$ yielding crystals suitable for diffraction studies. The crystallization process yields crystals of both the previously reported 1:1 co-crystal (Staun \& Oliver, 2015) and those of the title compound. Presumably the differences in solvent composition and time for crystallization can yield one polymorph over the other. Several crystallization attempts were made using the methodology described herein (slow evaporation from methanol) and all yielded mixtures of the
1:1 and the 3:1 co-crystals reported herein. No evidence of the Campos-Gaxiola structure was observed within the crystals examined (reported as colorless rectangular prisms).

\section{Refinement}

Crystal data, data collection and structure refinement details are summarized in Table 3. Where possible, hydrogen atoms were initially located from a difference Fourier map and were subsequently refined using a riding model with $\mathrm{C}-\mathrm{H}=0.95 \AA$, $\mathrm{N}-\mathrm{H}=0.88 \AA$ and $\mathrm{O}-\mathrm{H}=0.84 \AA$. $U_{\text {iso }}(\mathrm{H})$ was set to $1.2 U_{\text {eq }}(\mathrm{C} / \mathrm{N})$ and $1.5 U_{\text {eq }}(\mathrm{O})$. The reliability for the correct enantiomorph of the space group is low, due to the use of Mo $K \alpha$ radiation with a light atom structure. Analysis of the Flack $x$ [0.1 (10); Flack, 1983], Hooft y [0.2 (10); Hooft et al., 2008] and Parsons $z$ [-0.2 (12); Parsons et al., 2013] parameters tends to indicate that the correct enantiomorph of the space group and absolute structure has been determined (Flack \& Bernardinelli, 1999). Since these values are not close to zero the model could be refined as a racemic twin. However, this does not yield new or useful information and we have retained the standard model.

\section{References}

Bhogala, B. R., Basavoju, S. \& Nangia, A. (2005). CrystEngComm, 7, 551-562.

Bruker (2012). APEX2 and SAINT. Bruker AXS Inc., Madison, Wisconsin, USA.

Campos-Gaxiola, J. J., Zamora Falcon, F., Corral Higuera, R., Höpfl, H. \& Cruz-Enríquez, A. (2014). Acta Cryst. E70, o453-o454.

Castillo, O., Luque, A., Lloret, F. \& Román, P. (2001). Inorg. Chim. Acta, 324, 141-149.

Dolomanov, O. V., Bourhis, L. J., Gildea, R. J., Howard, J. A. K. \& Puschmann, H. (2009). J. Appl. Cryst. 42, 339-341.

Flack, H. D. (1983). Acta Cryst. A39, 876-881.

Flack, H. D. \& Bernardinelli, G. (1999). Acta Cryst. A55, 908-915.

Groom, C. R. \& Allen, F. H. (2014). Angew. Chem. Int. Ed. 53, 662671.

Hooft, R. W. W., Straver, L. H. \& Spek, A. L. (2008). J. Appl. Cryst. 41, 96-103.

Krause, L., Herbst-Irmer, R., Sheldrick, G. M. \& Stalke, D. (2015). J. Appl. Cryst. 48, 3-10.

Macrae, C. F., Bruno, I. J., Chisholm, J. A., Edgington, P. R., McCabe, P., Pidcock, E., Rodriguez-Monge, L., Taylor, R., van de Streek, J. \& Wood, P. A. (2008). J. Appl. Cryst. 41, 466-470.

Parsons, S., Flack, H. D. \& Wagner, T. (2013). Acta Cryst. B69, 249259.

Qian, J., Jiang, F., Zhang, L., Su, K., Pan, J., Li, Q., Yuan, D. \& Hong, M. (2014). Chem. Commun. 50, 1678-1681.

Shattock, T. R., Arora, K. K., Vishweshwar, P. \& Zaworotko, M. J. (2008). Cryst. Growth Des. 8, 4533-4545.

Sheldrick, G. M. (2008). Acta Cryst. A64, 112-122.

Sheldrick, G. M. (2015). Acta Cryst. C71, 3-8.

Spek, A. L. (2009). Acta Cryst. D65, 148-155.

Staun, S. L. \& Oliver, A. G. (2012). Acta Cryst. C68, o84-o87.

Staun, S. L. \& Oliver, A. G. (2015). Acta Cryst. E71, 861-863.

Tyl, A., Nowak, M. \& Kusz, J. (2008). Acta Cryst. C64, o661-o664. Westrip, S. P. (2010). J. Appl. Cryst. 43, 920-925. 


\section{supporting information}

Acta Cryst. (2015). E71, 1283-1286 [https://doi.org/10.1107/S2056989015017867]

\section{Crystal structure of benzene-1,3,5-tricarboxylic acid-4-pyridone (1/3)}

\section{Selena L. Staun and Allen G. Oliver}

\section{Computing details}

Data collection: APEX2 (Bruker, 2012); cell refinement: SAINT (Bruker 2012); data reduction: SAINT (Bruker 2012); program(s) used to solve structure: SHELXS97 (Sheldrick, 2008); program(s) used to refine structure: SHELXL2014/7 (Sheldrick, 2015); molecular graphics: OLEX2 (Dolomanov et al., 2009) and Mercury (Macrae et al., 2008); software used to prepare material for publication: publCIF (Westrip, 2010).

Benzene-1,3,5-tricarboxylic acid-4-pyridone (1/3)

Crystal data

$\mathrm{C}_{9} \mathrm{H}_{6} \mathrm{O}_{6} \cdot 3 \mathrm{C}_{5} \mathrm{H}_{5} \mathrm{NO}$

$M_{r}=495.44$

Orthorhombic, Pna2 ${ }_{1}$

$a=12.699$ (3) $\AA$

$b=26.498(6) \AA$

$c=6.6591(14) \AA$

$V=2240.9(8) \AA^{3}$

$Z=4$

$F(000)=1032$

\section{Data collection}

Bruker APEXII diffractometer

Radiation source: fine-focus sealed tube Graphite monochromator Detector resolution: 8.33 pixels $\mathrm{mm}^{-1}$ combination of $\omega$ and $\varphi$-scans Absorption correction: multi-scan

(SADABS; Krause et al., 2015)

$T_{\text {min }}=0.647, T_{\text {max }}=0.745$

\section{Refinement}

Refinement on $F^{2}$

Least-squares matrix: full

$R\left[F^{2}>2 \sigma\left(F^{2}\right)\right]=0.069$

$w R\left(F^{2}\right)=0.171$

$S=1.04$

3257 reflections

328 parameters

1 restraint

Primary atom site location: structure-invariant direct methods

Secondary atom site location: difference Fourier map
$D_{\mathrm{x}}=1.469 \mathrm{Mg} \mathrm{m}^{-3}$

Mo $K \alpha$ radiation, $\lambda=0.71073 \AA$

Cell parameters from 1078 reflections

$\theta=3.1-19.2^{\circ}$

$\mu=0.11 \mathrm{~mm}^{-1}$

$T=120 \mathrm{~K}$

Rod, colorless

$0.11 \times 0.07 \times 0.05 \mathrm{~mm}$

19034 measured reflections

3257 independent reflections

2418 reflections with $I>2 \sigma(I)$

$R_{\text {int }}=0.109$

$\theta_{\max }=23.4^{\circ}, \theta_{\min }=1.5^{\circ}$

$h=-14 \rightarrow 14$

$k=-29 \rightarrow 29$

$l=-7 \rightarrow 7$

Hydrogen site location: inferred from neighbouring sites

$\mathrm{H}$-atom parameters constrained

$w=1 /\left[\sigma^{2}\left(F_{\mathrm{o}}^{2}\right)+(0.1002 P)^{2}\right]$

where $P=\left(F_{\mathrm{o}}^{2}+2 F_{\mathrm{c}}^{2}\right) / 3$

$(\Delta / \sigma)_{\max }<0.001$

$\Delta \rho_{\max }=0.43$ e $\AA^{-3}$

$\Delta \rho_{\min }=-0.43$ e $\AA^{-3}$

Absolute structure: Flack $x$ determined using 801 quotients $\left[\left(I^{+}\right)-\left(I^{)}\right)\right] /\left[\left(I^{+}\right)+\left(I^{-}\right)\right]$(Parsons et al., 2013).

Absolute structure parameter: 0.1 (10) 


\section{Special details}

Geometry. All e.s.d.'s (except the e.s.d. in the dihedral angle between two 1.s. planes) are estimated using the full covariance matrix. The cell e.s.d.'s are taken into account individually in the estimation of e.s.d.'s in distances, angles and torsion angles; correlations between e.s.d.'s in cell parameters are only used when they are defined by crystal symmetry. An approximate (isotropic) treatment of cell e.s.d.'s is used for estimating e.s.d.'s involving l.s. planes.

Fractional atomic coordinates and isotropic or equivalent isotropic displacement parameters $\left(\AA^{2}\right)$

\begin{tabular}{|c|c|c|c|c|}
\hline & $x$ & $y$ & $z$ & $U_{\text {iso }} * / U_{\text {eq }}$ \\
\hline $\mathrm{O} 1$ & $0.4424(4)$ & $0.29680(18)$ & $0.8670(10)$ & $0.0258(14)$ \\
\hline N1 & $0.1387(5)$ & $0.2479(3)$ & 0.8807 (11) & $0.0282(18)$ \\
\hline $\mathrm{H} 1 \mathrm{~N}$ & 0.0730 & 0.2374 & 0.8841 & $0.034^{*}$ \\
\hline $\mathrm{C} 1$ & $0.2175(6)$ & $0.2147(3)$ & $0.9013(15)$ & $0.029(2)$ \\
\hline H1 & 0.2017 & 0.1799 & 0.9202 & $0.035^{*}$ \\
\hline $\mathrm{C} 2$ & $0.3205(6)$ & $0.2300(3)$ & 0.8955 (14) & $0.028(2)$ \\
\hline $\mathrm{H} 2$ & 0.3754 & 0.2059 & 0.9094 & $0.034^{*}$ \\
\hline $\mathrm{C} 3$ & $0.3455(5)$ & $0.2814(3)$ & $0.8692(14)$ & $0.023(2)$ \\
\hline $\mathrm{C} 4$ & $0.2598(6)$ & $0.3152(3)$ & $0.8499(14)$ & $0.029(2)$ \\
\hline $\mathrm{H} 4$ & 0.2720 & 0.3503 & 0.8335 & $0.035^{*}$ \\
\hline $\mathrm{C} 5$ & $0.1594(6)$ & 0.2968 & $0.8551(15)$ & $0.030(2)$ \\
\hline H5 & 0.1023 & 0.3197 & 0.8400 & $0.036^{*}$ \\
\hline $\mathrm{O} 2$ & $0.7873(4)$ & $0.71124(18)$ & $0.8498(10)$ & $0.0258(14)$ \\
\hline $\mathrm{N} 2$ & $1.1005(5)$ & $0.7401(2)$ & $0.8856(11)$ & $0.0268(18)$ \\
\hline $\mathrm{H} 2 \mathrm{~N}$ & 1.1684 & 0.7465 & 0.8913 & $0.032 *$ \\
\hline C6 & $1.0301(6)$ & $0.7782(3)$ & $0.9018(15)$ & $0.029(2)$ \\
\hline H6 & 1.0550 & 0.8116 & 0.9216 & $0.034^{*}$ \\
\hline $\mathrm{C} 7$ & $0.9253(6)$ & $0.7700(3)$ & 0.8907 (14) & $0.025(2)$ \\
\hline $\mathrm{H} 7$ & 0.8779 & 0.7976 & 0.9023 & $0.030 *$ \\
\hline $\mathrm{C} 8$ & $0.8859(5)$ & $0.7208(3)$ & 0.8619 (14) & $0.0216(19)$ \\
\hline $\mathrm{C} 9$ & $0.9618(5)$ & $0.6815(3)$ & $0.8493(14)$ & $0.026(2)$ \\
\hline H9 & 0.9392 & 0.6476 & 0.8330 & $0.031 *$ \\
\hline $\mathrm{C} 10$ & $1.0663(6)$ & $0.6920(3)$ & 0.8605 (14) & $0.028(2)$ \\
\hline H10 & 1.1161 & 0.6654 & 0.8507 & $0.034^{*}$ \\
\hline $\mathrm{O} 3$ & $0.0529(4)$ & $0.56842(19)$ & $0.8945(9)$ & $0.0217(14)$ \\
\hline N3 & $-0.0040(5)$ & $0.5697(3)$ & $1.4925(12)$ & $0.0283(19)$ \\
\hline $\mathrm{H} 3 \mathrm{~N}$ & -0.0161 & 0.5691 & 1.6226 & $0.034^{*}$ \\
\hline C11 & $-0.0812(6)$ & $0.5564(3)$ & $1.3647(15)$ & $0.023(2)$ \\
\hline H11 & -0.1479 & 0.5468 & 1.4167 & $0.028^{*}$ \\
\hline $\mathrm{C} 12$ & $-0.0659(7)$ & $0.5566(3)$ & $1.1636(14)$ & $0.025(2)$ \\
\hline H12 & -0.1218 & 0.5476 & 1.0756 & $0.030 *$ \\
\hline $\mathrm{C} 13$ & $0.0335(6)$ & $0.5702(3)$ & $1.0850(13)$ & $0.020(2)$ \\
\hline C14 & $0.1109(6)$ & $0.5847(3)$ & $1.2259(13)$ & $0.023(2)$ \\
\hline H13 & 0.1780 & 0.5954 & 1.1794 & $0.027 *$ \\
\hline $\mathrm{C} 15$ & $0.0916(7)$ & 0.5838 (3) & $1.4248(13)$ & $0.022(2)$ \\
\hline H15 & 0.1453 & 0.5931 & 1.5172 & $0.026^{*}$ \\
\hline $\mathrm{O} 4$ & $0.6262(4)$ & $0.39539(18)$ & $0.7880(11)$ & $0.0327(16)$ \\
\hline $\mathrm{O} 5$ & 0.4504 (3) & $0.39158(18)$ & $0.7983(10)$ & $0.0270(14)$ \\
\hline $\mathrm{H} 5 \mathrm{O}$ & 0.4625 & 0.3607 & 0.8143 & $0.040^{*}$ \\
\hline
\end{tabular}




$\begin{array}{lllll}\text { O6 } & 0.7915(4) & 0.57133(19) & 0.7531(10) & 0.0257(14) \\ \text { O7 } & 0.6792(4) & 0.63488(19) & 0.8051(13) & 0.046(2) \\ \text { H7O } & 0.7346 & 0.6513 & 0.8273 & 0.069^{*} \\ \text { O8 } & 0.2964(4) & 0.61537(19) & 0.7789(11) & 0.0293(14) \\ \text { O9 } & 0.2359(4) & 0.53621(19) & 0.8195(9) & 0.0261(15) \\ \text { H9O } & 0.1794 & 0.5512 & 0.8474 & 0.039^{*} \\ \text { C16 } & 0.5281(5) & 0.4719(3) & 0.7808(14) & 0.0161(17) \\ \text { C17 } & 0.6164(5) & 0.5025(3) & 0.7756(13) & 0.0196(19) \\ \text { H17 } & 0.6845 & 0.4878 & 0.7736 & 0.023^{*} \\ \text { C18 } & 0.6062(5) & 0.5544(3) & 0.7733(13) & 0.0163(17) \\ \text { C19 } & 0.5061(6) & 0.5761(3) & 0.7777(14) & 0.0200(18) \\ \text { H19 } & 0.4990 & 0.6118 & 0.7774 & 0.024^{*} \\ \text { C20 } & 0.4175(5) & 0.5460(3) & 0.7823(13) & 0.0177(18) \\ \text { C21 } & 0.4275(6) & 0.4933(3) & 0.7853(14) & 0.0195(18) \\ \text { H21 } & 0.3667 & 0.4725 & 0.7903 & 0.023^{*} \\ \text { C22 } & 0.5408(6) & 0.4164(3) & 0.7873(14) & 0.0229(19) \\ \text { C23 } & 0.3122(5) & 0.5701(3) & 0.7927(13) & 0.021(2) \\ \text { C24 } & 0.7028(6) & 0.5873(3) & 0.7757(14) & 0.0204(18) \\ \end{array}$

Atomic displacement parameters $\left(\AA^{2}\right)$

\begin{tabular}{lllllll}
\hline & $U^{11}$ & $U^{22}$ & $U^{33}$ & $U^{12}$ & $U^{13}$ & $U^{23}$ \\
\hline O1 & $0.011(3)$ & $0.021(3)$ & $0.046(4)$ & $-0.002(2)$ & $0.004(3)$ & $0.002(3)$ \\
N1 & $0.013(3)$ & $0.029(4)$ & $0.043(5)$ & $-0.010(3)$ & $0.004(4)$ & $-0.003(4)$ \\
C1 & $0.024(5)$ & $0.025(5)$ & $0.040(6)$ & $-0.008(4)$ & $-0.001(4)$ & $-0.001(5)$ \\
C2 & $0.024(5)$ & $0.024(5)$ & $0.037(6)$ & $0.002(4)$ & $0.005(4)$ & $0.001(4)$ \\
C3 & $0.016(4)$ & $0.024(4)$ & $0.028(5)$ & $0.001(4)$ & $-0.001(4)$ & $-0.008(4)$ \\
C4 & $0.021(4)$ & $0.026(5)$ & $0.039(6)$ & $-0.005(4)$ & $0.000(5)$ & $0.001(5)$ \\
C5 & $0.021(4)$ & $0.030(5)$ & $0.038(6)$ & $0.002(4)$ & $-0.002(4)$ & $0.001(5)$ \\
O2 & $0.013(3)$ & $0.022(3)$ & $0.042(4)$ & $0.000(2)$ & $-0.004(3)$ & $-0.003(3)$ \\
N2 & $0.012(3)$ & $0.030(4)$ & $0.038(5)$ & $-0.003(3)$ & $-0.003(4)$ & $0.002(4)$ \\
C6 & $0.018(5)$ & $0.021(5)$ & $0.046(6)$ & $-0.006(4)$ & $0.000(4)$ & $0.003(5)$ \\
C7 & $0.023(5)$ & $0.014(4)$ & $0.037(5)$ & $0.001(3)$ & $0.001(4)$ & $0.006(4)$ \\
C8 & $0.011(4)$ & $0.028(5)$ & $0.026(5)$ & $-0.005(3)$ & $-0.006(4)$ & $0.002(4)$ \\
C9 & $0.020(4)$ & $0.014(4)$ & $0.044(6)$ & $-0.003(3)$ & $0.002(4)$ & $0.001(4)$ \\
C10 & $0.021(4)$ & $0.025(5)$ & $0.040(6)$ & $0.000(4)$ & $0.004(4)$ & $0.001(5)$ \\
O3 & $0.018(3)$ & $0.025(3)$ & $0.023(4)$ & $0.004(2)$ & $0.001(3)$ & $0.000(3)$ \\
N3 & $0.042(5)$ & $0.019(4)$ & $0.024(4)$ & $0.003(4)$ & $0.001(4)$ & $-0.005(3)$ \\
C11 & $0.012(4)$ & $0.022(5)$ & $0.036(6)$ & $0.000(3)$ & $0.003(4)$ & $0.001(5)$ \\
C12 & $0.024(5)$ & $0.017(5)$ & $0.033(6)$ & $0.003(4)$ & $-0.003(4)$ & $-0.007(4)$ \\
C13 & $0.024(5)$ & $0.006(4)$ & $0.028(6)$ & $0.005(4)$ & $0.001(4)$ & $0.000(4)$ \\
C14 & $0.011(4)$ & $0.026(5)$ & $0.031(6)$ & $0.002(4)$ & $-0.002(4)$ & $0.000(4)$ \\
C15 & $0.018(5)$ & $0.024(5)$ & $0.022(5)$ & $0.001(4)$ & $-0.007(4)$ & $0.001(4)$ \\
O4 & $0.012(3)$ & $0.023(3)$ & $0.063(5)$ & $0.005(2)$ & $0.006(3)$ & $0.004(4)$ \\
O5 & $0.012(3)$ & $0.018(3)$ & $0.050(4)$ & $-0.003(2)$ & $0.000(3)$ & $0.006(4)$ \\
O6 & $0.009(3)$ & $0.025(3)$ & $0.043(4)$ & $0.000(2)$ & $0.001(3)$ & $-0.003(3)$ \\
O7 & $0.013(3)$ & $0.021(3)$ & $0.106(6)$ & $-0.004(2)$ & $0.011(4)$ & $-0.011(4)$ \\
O8 & $0.016(3)$ & $0.019(3)$ & $0.053(4)$ & $0.001(2)$ & $0.000(3)$ & $-0.004(3)$ \\
& & & & &
\end{tabular}




\begin{tabular}{lllllll}
\hline O9 & $0.013(3)$ & $0.022(3)$ & $0.043(4)$ & $-0.001(2)$ & $0.006(3)$ & $0.003(3)$ \\
C16 & $0.010(4)$ & $0.016(4)$ & $0.022(4)$ & $0.004(3)$ & $-0.003(4)$ & $-0.007(4)$ \\
C17 & $0.010(4)$ & $0.027(4)$ & $0.021(5)$ & $0.003(3)$ & $0.000(4)$ & $-0.003(4)$ \\
C18 & $0.011(4)$ & $0.015(4)$ & $0.022(4)$ & $0.002(3)$ & $0.002(4)$ & $-0.006(4)$ \\
C19 & $0.016(4)$ & $0.019(4)$ & $0.025(5)$ & $0.000(3)$ & $0.000(4)$ & $0.003(4)$ \\
C20 & $0.011(4)$ & $0.014(4)$ & $0.028(5)$ & $0.000(3)$ & $-0.003(4)$ & $-0.002(4)$ \\
C21 & $0.013(4)$ & $0.022(4)$ & $0.024(5)$ & $-0.003(3)$ & $0.001(4)$ & $0.004(4)$ \\
C22 & $0.021(5)$ & $0.023(4)$ & $0.025(5)$ & $0.000(4)$ & $0.003(4)$ & $-0.005(5)$ \\
C23 & $0.011(4)$ & $0.021(5)$ & $0.030(6)$ & $-0.005(3)$ & $-0.003(4)$ & $0.000(4)$ \\
C24 & $0.022(5)$ & $0.016(4)$ & $0.023(5)$ & $-0.001(3)$ & $0.002(4)$ & $0.002(4)$ \\
\hline
\end{tabular}

Geometric parameters $\left(\AA,{ }^{\circ}\right)$

\begin{tabular}{|c|c|c|c|}
\hline $\mathrm{O} 1-\mathrm{C} 3$ & $1.295(8)$ & C11-H11 & 0.9500 \\
\hline $\mathrm{N} 1-\mathrm{C} 5$ & $1.334(10)$ & $\mathrm{C} 12-\mathrm{C} 13$ & $1.413(12)$ \\
\hline $\mathrm{N} 1-\mathrm{C} 1$ & $1.340(10)$ & $\mathrm{C} 12-\mathrm{H} 12$ & 0.9500 \\
\hline $\mathrm{N} 1-\mathrm{H} 1 \mathrm{~N}$ & 0.8800 & $\mathrm{C} 13-\mathrm{C} 14$ & $1.413(12)$ \\
\hline $\mathrm{C} 1-\mathrm{C} 2$ & $1.371(11)$ & $\mathrm{C} 14-\mathrm{C} 15$ & $1.347(12)$ \\
\hline $\mathrm{C} 1-\mathrm{H} 1$ & 0.9500 & $\mathrm{C} 14-\mathrm{H} 13$ & 0.9500 \\
\hline $\mathrm{C} 2-\mathrm{C} 3$ & $1.410(11)$ & $\mathrm{C} 15-\mathrm{H} 15$ & 0.9500 \\
\hline $\mathrm{C} 2-\mathrm{H} 2$ & 0.9500 & $\mathrm{O} 4-\mathrm{C} 22$ & $1.220(8)$ \\
\hline $\mathrm{C} 3-\mathrm{C} 4$ & $1.414(10)$ & $\mathrm{O} 5-\mathrm{C} 22$ & $1.324(8)$ \\
\hline $\mathrm{C} 4-\mathrm{C} 5$ & $1.365(10)$ & $\mathrm{O} 5-\mathrm{H} 5 \mathrm{O}$ & 0.8400 \\
\hline $\mathrm{C} 4-\mathrm{H} 4$ & 0.9500 & $\mathrm{O} 6-\mathrm{C} 24$ & $1.213(9)$ \\
\hline $\mathrm{C} 5-\mathrm{H} 5$ & 0.9500 & $\mathrm{O} 7-\mathrm{C} 24$ & $1.310(9)$ \\
\hline $\mathrm{O} 2-\mathrm{C} 8$ & $1.280(8)$ & $\mathrm{O} 7-\mathrm{H} 7 \mathrm{O}$ & 0.8400 \\
\hline $\mathrm{N} 2-\mathrm{C} 6$ & $1.353(10)$ & $\mathrm{O} 8-\mathrm{C} 23$ & $1.221(8)$ \\
\hline $\mathrm{N} 2-\mathrm{C} 10$ & $1.356(10)$ & $\mathrm{O} 9-\mathrm{C} 23$ & $1.332(8)$ \\
\hline $\mathrm{N} 2-\mathrm{H} 2 \mathrm{~N}$ & 0.8800 & O9- $\mathrm{H} 9 \mathrm{O}$ & 0.8400 \\
\hline $\mathrm{C} 6-\mathrm{C} 7$ & $1.350(11)$ & $\mathrm{C} 16-\mathrm{C} 17$ & $1.385(9)$ \\
\hline $\mathrm{C} 6-\mathrm{H} 6$ & 0.9500 & $\mathrm{C} 16-\mathrm{C} 21$ & $1.398(9)$ \\
\hline $\mathrm{C} 7-\mathrm{C} 8$ & $1.410(10)$ & $\mathrm{C} 16-\mathrm{C} 22$ & $1.481(10)$ \\
\hline $\mathrm{C} 7-\mathrm{H} 7$ & 0.9500 & $\mathrm{C} 17-\mathrm{C} 18$ & $1.380(9)$ \\
\hline $\mathrm{C} 8-\mathrm{C} 9$ & $1.421(10)$ & C17-H17 & 0.9500 \\
\hline $\mathrm{C} 9-\mathrm{C} 10$ & $1.358(10)$ & $\mathrm{C} 18-\mathrm{C} 19$ & $1.395(10)$ \\
\hline C9-H9 & 0.9500 & $\mathrm{C} 18-\mathrm{C} 24$ & $1.506(10)$ \\
\hline $\mathrm{C} 10-\mathrm{H} 10$ & 0.9500 & $\mathrm{C} 19-\mathrm{C} 20$ & $1.380(10)$ \\
\hline $\mathrm{O} 3-\mathrm{C} 13$ & $1.293(10)$ & C19-H19 & 0.9500 \\
\hline N3-C11 & $1.345(10)$ & $\mathrm{C} 20-\mathrm{C} 21$ & $1.401(10)$ \\
\hline N3-C15 & $1.348(10)$ & $\mathrm{C} 20-\mathrm{C} 23$ & $1.484(10)$ \\
\hline $\mathrm{N} 3-\mathrm{H} 3 \mathrm{~N}$ & 0.8800 & $\mathrm{C} 21-\mathrm{H} 21$ & 0.9500 \\
\hline $\mathrm{C} 11-\mathrm{C} 12$ & $1.353(12)$ & & \\
\hline $\mathrm{C} 5-\mathrm{N} 1-\mathrm{C} 1$ & $120.3(7)$ & $\mathrm{C} 11-\mathrm{C} 12-\mathrm{C} 13$ & $119.7(8)$ \\
\hline $\mathrm{C} 5-\mathrm{N} 1-\mathrm{H} 1 \mathrm{~N}$ & 119.8 & $\mathrm{C} 11-\mathrm{C} 12-\mathrm{H} 12$ & 120.2 \\
\hline $\mathrm{C} 1-\mathrm{N} 1-\mathrm{H} 1 \mathrm{~N}$ & 119.8 & $\mathrm{C} 13-\mathrm{C} 12-\mathrm{H} 12$ & 120.2 \\
\hline $\mathrm{N} 1-\mathrm{C} 1-\mathrm{C} 2$ & $121.0(8)$ & $\mathrm{O} 3-\mathrm{C} 13-\mathrm{C} 12$ & $121.6(8)$ \\
\hline $\mathrm{N} 1-\mathrm{C} 1-\mathrm{H} 1$ & 119.5 & $\mathrm{O} 3-\mathrm{C} 13-\mathrm{C} 14$ & $121.9(8)$ \\
\hline
\end{tabular}




\begin{tabular}{|c|c|c|c|}
\hline $\mathrm{C} 2-\mathrm{C} 1-\mathrm{H} 1$ & 119.5 & $\mathrm{C} 12-\mathrm{C} 13-\mathrm{C} 14$ & $116.4(8)$ \\
\hline $\mathrm{C} 1-\mathrm{C} 2-\mathrm{C} 3$ & $120.4(7)$ & $\mathrm{C} 15-\mathrm{C} 14-\mathrm{C} 13$ & $121.4(9)$ \\
\hline $\mathrm{C} 1-\mathrm{C} 2-\mathrm{H} 2$ & 119.8 & $\mathrm{C} 15-\mathrm{C} 14-\mathrm{H} 13$ & 119.3 \\
\hline $\mathrm{C} 3-\mathrm{C} 2-\mathrm{H} 2$ & 119.8 & $\mathrm{C} 13-\mathrm{C} 14-\mathrm{H} 13$ & 119.3 \\
\hline $\mathrm{O} 1-\mathrm{C} 3-\mathrm{C} 2$ & $121.3(7)$ & $\mathrm{C} 14-\mathrm{C} 15-\mathrm{N} 3$ & $119.8(8)$ \\
\hline $\mathrm{O} 1-\mathrm{C} 3-\mathrm{C} 4$ & $122.0(7)$ & $\mathrm{C} 14-\mathrm{C} 15-\mathrm{H} 15$ & 120.1 \\
\hline $\mathrm{C} 2-\mathrm{C} 3-\mathrm{C} 4$ & $116.7(7)$ & $\mathrm{N} 3-\mathrm{C} 15-\mathrm{H} 15$ & 120.1 \\
\hline $\mathrm{C} 5-\mathrm{C} 4-\mathrm{C} 3$ & $119.4(7)$ & $\mathrm{C} 22-\mathrm{O} 5-\mathrm{H} 5 \mathrm{O}$ & 109.5 \\
\hline $\mathrm{C} 5-\mathrm{C} 4-\mathrm{H} 4$ & 120.3 & $\mathrm{C} 24-\mathrm{O} 7-\mathrm{H} 7 \mathrm{O}$ & 109.5 \\
\hline $\mathrm{C} 3-\mathrm{C} 4-\mathrm{H} 4$ & 120.3 & $\mathrm{C} 23-\mathrm{O} 9-\mathrm{H} 9 \mathrm{O}$ & 109.5 \\
\hline $\mathrm{N} 1-\mathrm{C} 5-\mathrm{C} 4$ & $122.2(7)$ & $\mathrm{C} 17-\mathrm{C} 16-\mathrm{C} 21$ & $120.2(6)$ \\
\hline $\mathrm{N} 1-\mathrm{C} 5-\mathrm{H} 5$ & 118.9 & $\mathrm{C} 17-\mathrm{C} 16-\mathrm{C} 22$ & $119.7(6)$ \\
\hline $\mathrm{C} 4-\mathrm{C} 5-\mathrm{H} 5$ & 118.9 & $\mathrm{C} 21-\mathrm{C} 16-\mathrm{C} 22$ & $120.1(6)$ \\
\hline $\mathrm{C} 6-\mathrm{N} 2-\mathrm{C} 10$ & $120.0(6)$ & $\mathrm{C} 18-\mathrm{C} 17-\mathrm{C} 16$ & $120.5(7)$ \\
\hline $\mathrm{C} 6-\mathrm{N} 2-\mathrm{H} 2 \mathrm{~N}$ & 120.0 & $\mathrm{C} 18-\mathrm{C} 17-\mathrm{H} 17$ & 119.7 \\
\hline $\mathrm{C} 10-\mathrm{N} 2-\mathrm{H} 2 \mathrm{~N}$ & 120.0 & $\mathrm{C} 16-\mathrm{C} 17-\mathrm{H} 17$ & 119.7 \\
\hline $\mathrm{C} 7-\mathrm{C} 6-\mathrm{N} 2$ & $121.8(8)$ & $\mathrm{C} 17-\mathrm{C} 18-\mathrm{C} 19$ & $119.7(7)$ \\
\hline $\mathrm{C} 7-\mathrm{C} 6-\mathrm{H} 6$ & 119.1 & $\mathrm{C} 17-\mathrm{C} 18-\mathrm{C} 24$ & $120.0(6)$ \\
\hline $\mathrm{N} 2-\mathrm{C} 6-\mathrm{H} 6$ & 119.1 & $\mathrm{C} 19-\mathrm{C} 18-\mathrm{C} 24$ & $120.2(6)$ \\
\hline $\mathrm{C} 6-\mathrm{C} 7-\mathrm{C} 8$ & $120.4(7)$ & $\mathrm{C} 20-\mathrm{C} 19-\mathrm{C} 18$ & $120.3(7)$ \\
\hline $\mathrm{C} 6-\mathrm{C} 7-\mathrm{H} 7$ & 119.8 & $\mathrm{C} 20-\mathrm{C} 19-\mathrm{H} 19$ & 119.8 \\
\hline $\mathrm{C} 8-\mathrm{C} 7-\mathrm{H} 7$ & 119.8 & $\mathrm{C} 18-\mathrm{C} 19-\mathrm{H} 19$ & 119.8 \\
\hline $\mathrm{O} 2-\mathrm{C} 8-\mathrm{C} 7$ & $122.6(7)$ & $\mathrm{C} 19-\mathrm{C} 20-\mathrm{C} 21$ & $120.2(6)$ \\
\hline $\mathrm{O} 2-\mathrm{C} 8-\mathrm{C} 9$ & $121.0(7)$ & $\mathrm{C} 19-\mathrm{C} 20-\mathrm{C} 23$ & $119.2(6)$ \\
\hline $\mathrm{C} 7-\mathrm{C} 8-\mathrm{C} 9$ & $116.4(7)$ & $\mathrm{C} 21-\mathrm{C} 20-\mathrm{C} 23$ & $120.6(6)$ \\
\hline $\mathrm{C} 10-\mathrm{C} 9-\mathrm{C} 8$ & $120.6(7)$ & $\mathrm{C} 16-\mathrm{C} 21-\mathrm{C} 20$ & $119.1(6)$ \\
\hline $\mathrm{C} 10-\mathrm{C} 9-\mathrm{H} 9$ & 119.7 & $\mathrm{C} 16-\mathrm{C} 21-\mathrm{H} 21$ & 120.4 \\
\hline $\mathrm{C} 8-\mathrm{C} 9-\mathrm{H} 9$ & 119.7 & $\mathrm{C} 20-\mathrm{C} 21-\mathrm{H} 21$ & 120.4 \\
\hline $\mathrm{N} 2-\mathrm{C} 10-\mathrm{C} 9$ & $120.8(7)$ & $\mathrm{O} 4-\mathrm{C} 22-\mathrm{O} 5$ & $123.0(7)$ \\
\hline $\mathrm{N} 2-\mathrm{C} 10-\mathrm{H} 10$ & 119.6 & $\mathrm{O} 4-\mathrm{C} 22-\mathrm{C} 16$ & $123.4(7)$ \\
\hline $\mathrm{C} 9-\mathrm{C} 10-\mathrm{H} 10$ & 119.6 & $\mathrm{O} 5-\mathrm{C} 22-\mathrm{C} 16$ & $113.6(6)$ \\
\hline $\mathrm{C} 11-\mathrm{N} 3-\mathrm{C} 15$ & $121.2(8)$ & $\mathrm{O} 8-\mathrm{C} 23-\mathrm{O} 9$ & $123.6(6)$ \\
\hline $\mathrm{C} 11-\mathrm{N} 3-\mathrm{H} 3 \mathrm{~N}$ & 119.4 & $\mathrm{O} 8-\mathrm{C} 23-\mathrm{C} 20$ & $124.6(6)$ \\
\hline $\mathrm{C} 15-\mathrm{N} 3-\mathrm{H} 3 \mathrm{~N}$ & 119.4 & $\mathrm{O} 9-\mathrm{C} 23-\mathrm{C} 20$ & $111.8(6)$ \\
\hline $\mathrm{N} 3-\mathrm{C} 11-\mathrm{C} 12$ & $121.4(8)$ & $\mathrm{O} 6-\mathrm{C} 24-\mathrm{O} 7$ & $124.5(7)$ \\
\hline $\mathrm{N} 3-\mathrm{C} 11-\mathrm{H} 11$ & 119.3 & $\mathrm{O} 6-\mathrm{C} 24-\mathrm{C} 18$ & $123.6(7)$ \\
\hline $\mathrm{C} 12-\mathrm{C} 11-\mathrm{H} 11$ & 119.3 & $\mathrm{O} 7-\mathrm{C} 24-\mathrm{C} 18$ & $111.9(6)$ \\
\hline $\mathrm{C} 5-\mathrm{N} 1-\mathrm{C} 1-\mathrm{C} 2$ & $-0.3(15)$ & $\mathrm{C} 21-\mathrm{C} 16-\mathrm{C} 17-\mathrm{C} 18$ & $-0.5(14)$ \\
\hline $\mathrm{N} 1-\mathrm{C} 1-\mathrm{C} 2-\mathrm{C} 3$ & $0.5(15)$ & $\mathrm{C} 22-\mathrm{C} 16-\mathrm{C} 17-\mathrm{C} 18$ & $-178.8(8)$ \\
\hline $\mathrm{C} 1-\mathrm{C} 2-\mathrm{C} 3-\mathrm{O} 1$ & $178.7(9)$ & $\mathrm{C} 16-\mathrm{C} 17-\mathrm{C} 18-\mathrm{C} 19$ & $0.4(13)$ \\
\hline $\mathrm{C} 1-\mathrm{C} 2-\mathrm{C} 3-\mathrm{C} 4$ & $0.1(14)$ & $\mathrm{C} 16-\mathrm{C} 17-\mathrm{C} 18-\mathrm{C} 24$ & $177.7(8)$ \\
\hline $\mathrm{O} 1-\mathrm{C} 3-\mathrm{C} 4-\mathrm{C} 5$ & $-179.4(9)$ & $\mathrm{C} 17-\mathrm{C} 18-\mathrm{C} 19-\mathrm{C} 20$ & $-0.6(14)$ \\
\hline $\mathrm{C} 2-\mathrm{C} 3-\mathrm{C} 4-\mathrm{C} 5$ & $-0.8(14)$ & $\mathrm{C} 24-\mathrm{C} 18-\mathrm{C} 19-\mathrm{C} 20$ & $-177.8(8)$ \\
\hline $\mathrm{C} 1-\mathrm{N} 1-\mathrm{C} 5-\mathrm{C} 4$ & $-0.4(15)$ & $\mathrm{C} 18-\mathrm{C} 19-\mathrm{C} 20-\mathrm{C} 21$ & $0.8(14)$ \\
\hline $\mathrm{C} 3-\mathrm{C} 4-\mathrm{C} 5-\mathrm{N} 1$ & $1.0(15)$ & $\mathrm{C} 18-\mathrm{C} 19-\mathrm{C} 20-\mathrm{C} 23$ & $178.3(8)$ \\
\hline $\mathrm{C} 10-\mathrm{N} 2-\mathrm{C} 6-\mathrm{C} 7$ & $-1.1(14)$ & $\mathrm{C} 17-\mathrm{C} 16-\mathrm{C} 21-\mathrm{C} 20$ & $0.7(13)$ \\
\hline $\mathrm{N} 2-\mathrm{C} 6-\mathrm{C} 7-\mathrm{C} 8$ & $0.2(15)$ & $\mathrm{C} 22-\mathrm{C} 16-\mathrm{C} 21-\mathrm{C} 20$ & $179.0(9)$ \\
\hline
\end{tabular}




$\begin{array}{llll}\mathrm{C} 6-\mathrm{C} 7-\mathrm{C} 8-\mathrm{O} 2 & -179.8(9) & \mathrm{C} 19-\mathrm{C} 20-\mathrm{C} 21-\mathrm{C} 16 & -0.9(13) \\ \mathrm{C} 6-\mathrm{C} 7-\mathrm{C} 8-\mathrm{C} 9 & 1.0(14) & \mathrm{C} 23-\mathrm{C} 20-\mathrm{C} 21-\mathrm{C} 16 & -178.3(8) \\ \mathrm{O} 2-\mathrm{C} 8-\mathrm{C} 9-\mathrm{C} 10 & 179.4(9) & \mathrm{C} 17-\mathrm{C} 16-\mathrm{C} 22-\mathrm{O} 4 & -0.2(15) \\ \mathrm{C} 7-\mathrm{C} 8-\mathrm{C} 9-\mathrm{C} 10 & -1.4(14) & \mathrm{C} 21-\mathrm{C} 16-\mathrm{C} 22-\mathrm{O} 4 & -178.4(9) \\ \mathrm{C} 6-\mathrm{N} 2-\mathrm{C} 10-\mathrm{C} 9 & 0.7(14) & \mathrm{C} 17-\mathrm{C} 16-\mathrm{C} 22-\mathrm{O} 5 & 177.9(7) \\ \mathrm{C} 8-\mathrm{C} 9-\mathrm{C} 10-\mathrm{N} 2 & 0.6(15) & \mathrm{C} 21-\mathrm{C} 16-\mathrm{C} 22-\mathrm{O} 5 & -0.3(13) \\ \mathrm{C} 15-\mathrm{N} 3-\mathrm{C} 11-\mathrm{C} 12 & 0.2(13) & \mathrm{C} 19-\mathrm{C} 20-\mathrm{C} 23-\mathrm{O} 8 & 7.1(15) \\ \mathrm{N} 3-\mathrm{C} 11-\mathrm{C} 12-\mathrm{C} 13 & 1.0(14) & \mathrm{C} 21-\mathrm{C} 20-\mathrm{C} 23-\mathrm{O} 8 & -175.5(9) \\ \mathrm{C} 11-\mathrm{C} 12-\mathrm{C} 13-\mathrm{O} 3 & 176.6(8) & \mathrm{C} 19-\mathrm{C} 20-\mathrm{C} 23-\mathrm{O} 9 & -172.7(8) \\ \mathrm{C} 11-\mathrm{C} 12-\mathrm{C} 13-\mathrm{C} 14 & -2.2(13) & \mathrm{C} 21-\mathrm{C} 20-\mathrm{C} 23-\mathrm{O} 9 & 4.7(12) \\ \mathrm{O} 3-\mathrm{C} 13-\mathrm{C} 14-\mathrm{C} 15 & -176.3(8) & \mathrm{C} 17-\mathrm{C} 18-\mathrm{C} 24-\mathrm{O} 6 & 10.1(14) \\ \mathrm{C} 12-\mathrm{C} 13-\mathrm{C} 14-\mathrm{C} 15 & 2.4(13) & \mathrm{C} 19-\mathrm{C} 18-\mathrm{C} 24-\mathrm{O} 6 & -172.7(9) \\ \mathrm{C} 13-\mathrm{C} 14-\mathrm{C} 15-\mathrm{N} 3 & -1.3(14) & \mathrm{C} 17-\mathrm{C} 18-\mathrm{C} 24-\mathrm{O} 7 & -169.9(8) \\ \mathrm{C} 11-\mathrm{N} 3-\mathrm{C} 15-\mathrm{C} 14 & -0.1(13) & \mathrm{C} 19-\mathrm{C} 18-\mathrm{C} 24-\mathrm{O} 7 & 7.3(12)\end{array}$

Hydrogen-bond geometry $\left(\AA,{ }^{\circ}\right)$

\begin{tabular}{|c|c|c|c|c|}
\hline$D-\mathrm{H} \cdots A$ & $D-\mathrm{H}$ & $\mathrm{H} \cdots A$ & $D \cdots A$ & $D-\mathrm{H} \cdots A$ \\
\hline $\mathrm{N} 1-\mathrm{H} 1 N \cdots \mathrm{O} 1^{\mathrm{i}}$ & 0.88 & 1.89 & $2.762(8)$ & 169 \\
\hline $\mathrm{N} 2-\mathrm{H} 2 N \cdots \mathrm{O} 2^{\mathrm{ii}}$ & 0.88 & 1.90 & $2.711(8)$ & 152 \\
\hline $\mathrm{N} 3-\mathrm{H} 3 N \cdots \mathrm{O} 3^{\mathrm{iii}}$ & 0.88 & 2.01 & $2.773(10)$ & 144 \\
\hline $\mathrm{N} 3-\mathrm{H} 3 N \cdots \mathrm{O}^{\mathrm{iv}}$ & 0.88 & 2.59 & $3.124(9)$ & 120 \\
\hline $\mathrm{O} 5-\mathrm{H} 5 \mathrm{O} \cdots \mathrm{O} 1$ & 0.84 & 1.75 & $2.555(7)$ & 161 \\
\hline $\mathrm{O} 7-\mathrm{H} 7 \mathrm{O}^{\cdots} \mathrm{O} 2$ & 0.84 & 1.73 & $2.463(7)$ & 145 \\
\hline $\mathrm{O} 9-\mathrm{H} 9 O \cdots \mathrm{O} 3$ & 0.84 & 1.70 & $2.526(7)$ & 167 \\
\hline $\mathrm{C} 1-\mathrm{H} 1 \cdots \mathrm{O} 4^{\mathrm{i}}$ & 0.95 & 2.38 & $3.227(10)$ & 148 \\
\hline $\mathrm{C} 4-\mathrm{H} 4 \cdots \mathrm{O} 5$ & 0.95 & 2.53 & $3.174(9)$ & 126 \\
\hline $\mathrm{C} 6-\mathrm{H} 6 \cdots \mathrm{O}^{\mathrm{ii}}$ & 0.95 & 2.26 & $3.051(9)$ & 140 \\
\hline $\mathrm{C} 7-\mathrm{H} 7 \cdots \mathrm{O} 8^{\mathrm{ii}}$ & 0.95 & 2.66 & $3.530(9)$ & 153 \\
\hline $\mathrm{C} 9-\mathrm{H} 9 \cdots \mathrm{O}^{v}$ & 0.95 & 2.58 & $3.227(9)$ & 126 \\
\hline $\mathrm{C} 11-\mathrm{H} 11 \cdots \mathrm{O} 6^{\mathrm{iv}}$ & 0.95 & 2.46 & $3.076(11)$ & 123 \\
\hline $\mathrm{C} 11-\mathrm{H} 11 \cdots \mathrm{O}^{\mathrm{vi}}$ & 0.95 & 2.55 & $3.159(9)$ & 122 \\
\hline $\mathrm{C} 12-\mathrm{H} 12 \cdots \mathrm{O} 6^{\mathrm{vii}}$ & 0.95 & 2.49 & $3.302(11)$ & 143 \\
\hline $\mathrm{C} 14-\mathrm{H} 13 \cdots \mathrm{O} 4^{\text {viii }}$ & 0.95 & 2.60 & $3.405(10)$ & 143 \\
\hline $\mathrm{C} 15-\mathrm{H} 15 \cdots \mathrm{O} 8^{\mathrm{iii}}$ & 0.95 & 2.66 & $3.608(10)$ & 178 \\
\hline
\end{tabular}

Symmetry codes: (i) $x-1 / 2,-y+1 / 2, z$; (ii) $x+1 / 2,-y+3 / 2, z$; (iii) $x, y, z+1$; (iv) $x-1, y, z+1$; (v) $x+1, y, z$; (vi) $-x,-y+1, z+1 / 2$; (vii) $x-1, y, z$; (viii) $-x+1$, $-y+1, z+1 / 2$. 